\title{
Speculation of the Time-Dependent Change of FIB4 Index in Patients with Nonalcoholic Fatty Liver Disease: A Retrospective Study
}

\author{
Hiroshi Miyata $(\mathbb{D})$ and Satoru Miyata \\ Sugimoto Clinic, 3-2-27 Yamanouchi, Sumiyoshi-ku, Osaka city, Osaka-fu 558-0023, Japan \\ Correspondence should be addressed to Hiroshi Miyata; czx07100@nifty.com
}

Received 25 October 2017; Accepted 22 January 2018; Published 12 March 2018

Academic Editor: Branka Filipović

Copyright ( $(2018$ Hiroshi Miyata and Satoru Miyata. This is an open access article distributed under the Creative Commons Attribution License, which permits unrestricted use, distribution, and reproduction in any medium, provided the original work is properly cited.

\begin{abstract}
Aim. To speculate on the time-dependent change of FIB4 index in patients with nonalcoholic fatty liver disease (NAFLD) and its increase-decrease rate per year, simply and accurately. Methods. In all 23 patients with NAFLD with the value of FIB4 index over 1.30 at the peak, the period from the first to each examination date was calculated and this period (years) was regarded as $x$. Next, the mean value of FIB4 index during the past year to each examination date was regarded as $y$. In every $y$, the minimum and the maximum $y$ value were found out. Between $x$ corresponding to this minimum $y$ and $x$ corresponding to this maximum $y$, the correlation between $x$ and $y$ was analyzed as the main subject. Then, the slope of each correlation was investigated, because it should indicate increase-decrease rate per year. Results. In all 23 patients, the correlations as the main subject were recognized and the mean absolute value of correlation coefficient $(r)$ was $0.91 \pm 0.08$. As for the slope, the mean absolute value was $0.1371 \pm 0.1147$ (/year). Conclusion. Simply and accurately, the time-dependent change of FIB4 index and its increase-decrease rate per year could be approximately speculated.
\end{abstract}

\section{Introduction}

Nonalcoholic fatty liver disease (NAFLD) is one of the most common causes of chronic liver disease worldwide [1-5]. A liver biopsy still remains the gold standard for the diagnosis of nonalcoholic steatohepatitis (NASH), but it is difficult to perform liver biopsies in all patients with NAFLD. Therefore many noninvasive methods for estimating liver fibrosis have been developed; these are direct markers and the scoring systems, such as type IV collagen $7 \mathrm{~S}[6,7]$, hyaluronic acid $[8,9]$, aspartate aminotransferase (AST)/alanine aminotransferase (ALT) ratio (AAR) [9, 10], NAFLD fibrosis score [11], BARD score [12], NAFIC score [7], and so on.

FIB4 index has been developed to predict liver fibrosis in patients with HIV/HCV coinfection [13] and it is also useful for estimating liver fibrosis in patients with NAFLD [14-16]. However there were few reports analyzing the transition of FIB4 index during all the clinical period in patients with NAFLD. Probably for the dispersion of the data, it has been difficult to estimate the accurate value.
In this study the correlation between the period from the first to each examination date and the mean value of FIB4 index during the past year to each examination date was analyzed. This correlation was thought to be the timedependent change of the mean FIB4 index during the past one year and in the present study the correlation was proved to be extremely strong. Moreover, increase-decrease rate per year could be derived from the slope of the correlation in the scatter diagram.

In this retrospective study, the aim was to speculate approximately on the time-dependent change of FIB4 index and its increase-decrease rate per year, simply and accurately.

\section{Methods}

2.1. Patients. A total of 23 patients between October 1999 and June 2017 were enrolled with the following criteria: negative HBs antigen, negative HCV antibody, and negative antimitochondrial antibody [17]. Serum CRP levels were continuously negative. Patients whose values of anti-nuclear antibody 
(ANA) showed more than 1:160 were excluded [18]. The peak ALT levels were over 40 (U/L) for males or over $30(\mathrm{U} / \mathrm{L})$ for females [19-21]. The peak value of FIB4 index was over $1.30[14,15,22]$ in every patient. Fatty liver was diagnosed with ultrasonography and/or computed tomography. Drug induced liver injury and hereditary liver diseases were denied by the interview. Patients who consumed alcohol over $30 \mathrm{~g}$ per day for males or over $20 \mathrm{~g}$ per day for females were excluded $[14,23,24]$. Patients whose observed period in the clinic was less than two years were excluded. Finally patients whose maximum interval between examinations was more than one year were excluded.

All procedures in this study were conducted with the declaration of Helsinki (1964). The written informed consent was not applicable, because this is a retrospective study. In this study, direct data of AST, ALT, age, and platelet count were only used in patient characteristics and it was not possible to identify individuals.

\subsection{Correlations}

2.2.1. The Main Correlations. First, the period from the first to each examination date was calculated and this period (years) and was regarded as $x$. Next, the mean value of FIB4 index during the past one year to each examination date (the mean FIB4 index YTD) was regarded as $y$. Because of using the mean value during the past one year as $y, x$ less than 1.00 (years) and $y$ corresponding to this $x$ were excluded; the minimum $x$ value in every $x$ was more than 1.00 (years). In every $y$, the minimum $y$ value and the maximum $y$ value were found out. Between $x$ corresponding to this minimum $y$ and $x$ corresponding to this maximum $y$, the correlation between $x$ and $y$ was analyzed in every patient. This correlation was defined as the main correlation. There are two possibilities; either the values of correlation coefficient $(r)$ are positive or these are negative. The group with positive value of $r$ was defined as FIB4 index-increasing group and the group with negative value of $r$ was also defined as FIB4 index-decreasing group.

2.2.2. The After-Main Correlations. Then, another correlation was analyzed, except for the data during the period of the main correlation. However, both ends of the data in the main correlation were not excluded.

After the period of the main correlation, it was checked whether the period to the last examination date was more than 1.00 (years) or not. Only when this period was more than one year, the analysis was performed. The first data of this analysis was automatically the last data in the main correlation. In FIB4 index-increasing group, the minimum $y$ value was newly found out in this period. Yet, in FIB4 indexdecreasing group, the maximum $y$ value was newly found out. In both groups, from the maximum $x$ in the main correlation to $x$ corresponding to $y$ newly found out, the correlation between $x$ and $y$ was analyzed. This correlation was defined as the after-main correlation.

2.2.3. The Before-Main Correlations. Finally, before the period of the main correlation, it was checked whether the period from the first examination date was more than 2.00 (years) or not, because $x$ less than 1.00 (years) had been excluded. Only when this period was more than two years, the analysis was performed. The last data of this analysis was automatically the first data in the main correlation. In FIB4 index-increasing group, the maximum $y$ value was newly found out in this period. Yet in FIB4 index-decreasing group, the minimum $y$ value was newly found out. In both groups, from $x$ corresponding to $y$ newly found out to the minimum $x$ in the main correlation, the correlation between $x$ and $y$ was analyzed. This correlation was defined as the before-main correlation.

2.2.4. A Total of the Correlations Recognized in the Study. The cumulative correlations recognized in this study were shown.

2.3. Slopes of Correlations. In every patient, increase-decrease rate per year of the mean FIB4 index YTD was derived from the slope of the main correlation. In the same way it was also derived from each slope of the after-main correlation and/or the before-main correlation, if these correlations were recognized.

2.4. Statistics Analysis. Each correlation between two parameters was evaluated by Pearson's correlation. A $p$ value $(p)$ less than 0.05 was considered statistically significant. It was conducted by Microsoft Excel for MAC 2011.

\section{Results}

3.1. Patient Characteristics. 12 out of 23 patients (52.2\%) were male. In 20 patients the values of ANA were less than 1:40 [18] and in three patients these were $1: 40,1: 40$, and $1: 160$, respectively, and the values of anti-smooth muscle antibody were all less than 1:40 and also immunoglobulin G levels were all within the upper normal limit of the clinic [25]. In 17 patients computed tomography scans were performed. In all patients, the mean value of the peak ALT levels was $72 \pm 35$ $(\mathrm{U} / \mathrm{L})$ and that of the peak value of FIB4 index was 2.84 \pm 1.34 . In 10 patients the peak values of FIB4 index were more than $2.67[14,21]$, yet in nine patients those were less than 2.00. In all patients, the mean value of platelet count at the bottom was $165 \pm 45\left(\times 10^{9} / \mathrm{L}\right)$. Of 23 patients, 19 consumed no alcohol and the remaining four were all males (Table 1$)$.

\subsection{Correlations}

3.2.1. The Main Correlations. In all 23 patients the main correlations were recognized (Figure 1 ) and the mean absolute value of $r$ was $0.91 \pm 0.08$ (Table 2). Each $p$ was shown in Table 2. Of 23 patients, 17 were categorized in FIB4 indexincreasing group and the mean value of $r$ was $0.90 \pm 0.09$ ( 0.69 to 0.99 ). In 11 of these 17 , the values of $r$ were more than 0.90 . On the other hand, six of 23 patients were categorized in FIB4 index-decreasing group and the mean value of $r$ was $-0.94 \pm 0.02(-0.97$ to -0.91$)$. In all these six patients, the absolute values of $r$ were more than 0.90 . Therefore, in 17 out of 23 patients, the absolute values of $r$ were more than 0.90 . In a total of 23 patients, the mean value of interval between 
TABLE 1: Characteristics of all 23 patients.

\begin{tabular}{lccc}
\hline Patients $(n=23)$ & \multicolumn{2}{c}{$\begin{array}{c}\text { Laboratory findings } \\
\text { Peak value }\end{array}$} & $\begin{array}{c}\text { At the last examination } \\
\text { At the first examination }\end{array}$ \\
\hline Gender (male) & $58.2 \pm 8.5$ & $12(52.2 \%)$ & $68.8 \pm 9.5$ \\
Age (years) & $40 \pm 26$ & NA & $28 \pm 10$ \\
AST (U/L) & $49 \pm 35$ & $72 \pm 30$ & $26 \pm 12$ \\
ALT (U/L) & NA & $94 \pm 83$ & $45 \pm 44$ \\
GGT (U/L) & $1.66 \pm 0.78$ & $2.84 \pm 1.34$ & $2.04 \pm 0.82$ \\
FIB4 index & $0.88 \pm 0.22$ & $1.52 \pm 0.34$ & $1.17 \pm 0.31$ \\
AAR & $215 \pm 68$ & $165 \pm 45$ & $207 \pm 63$ \\
Platelet count $\left(\times 10^{9} / \mathrm{L}\right)$ & $\mathrm{NA}$ & $5.2 \pm 2.0$ & $4.4 \pm 1.4$ \\
Type IV collagen $7 \mathrm{~S}(\mathrm{ng} / \mathrm{mL})$ & $\mathrm{NA}$ & $1.09 \pm 0.86$ & $0.88 \pm 0.74$ \\
M2BPGi & & &
\end{tabular}

Mean \pm SD

Continuous variables were shown as mean \pm standard deviation. At the last examination both type IV collagen $7 \mathrm{~S}$ and M2BPGi were examined in all 23 patients. $n$, number of patients; NA, no analysis; AST, aspartate aminotransferase; ALT, alanine aminotransferase; GGT, gamma-glutamyl transpeptidase; AAR, AST/ALT ratio; M2BPGi, Mac-2 binding protein.

examinations was $0.17 \pm 0.09$ (years), that is, $64 \pm 33$ (days), and the mean value of the total clinical period was $10.7 \pm 4.6$ (years) (Table 2). Since $x$ less than 1.00 (years) were excluded, the total analyzed period was $9.5 \pm 4.5$ (years) (Table 2 ). The period in which the main correlation was recognized (the main correlation's period) was $6.6 \pm 4.5$ (years) and the mean ratio of the main correlation's period to the total analyzed period was $64 \pm 23 \%$ (27\% to $98 \%)$.

3.2.2. The After-Main Correlations. In 11 out of all 23 patients, each period to the last examination after the main correlation was more than 1.00 (years). In eight of these 11, the aftermain correlations were seen. The mean absolute value of $r$ was $0.93 \pm 0.04$ and each $p$ was shown in Table 3 . In the remaining three of these 11 , that is, in patients of cases 5,16 , and 18 , the correlations were not recognized statistically. In these three patients, numbers of analyzed data were four, five, and five, respectively, and the correlations were not recognized by $p=$ 0.17 and $r=-0.83$, by $p=0.09$ and $r=-0.82$, and by $p=0.07$ and $r=0.85$, respectively (Table 3 ).

3.2.3. The Before-Main Correlations. In 14 out of all 23 patients, each period from the first examination before the main correlation was more than 2.00 (years). In 10 of these 14 , the before-main correlations were seen. The mean absolute value of $r$ was $0.95 \pm 0.05$ and each $p$ was shown in Table 3 . In the remaining four of these 14 , that is, in patients of cases 6,8 , 11 , and 20 , the correlations were not recognized statistically. In two patients of cases 6 and 11, numbers of analyzed data for the correlations were both two and it was impossible to analyze. In the remaining two patients of cases 8 and 20 , numbers of analyzed data were three and four and the correlations were not recognized by $p=0.34$ and $r=-0.86$ and by $p=0.27$ and $r=0.73$, respectively (Table 3 ).

3.2.4. A Total of the Correlations Recognized in the Study. The cumulative number of all correlations recognized in this study was 41 (Table 3 ). The mean absolute value of $r$ was $0.92 \pm 0.07$. In 32 of 41 correlations the absolute values of $r$ were over 0.90 and in only three of 41 they were less than 0.80 (0.688 to 0.799$)$.

3.3. Slopes of Correlations. In all 23 main correlations, the values of increase-decrease rate per year of the mean FIB4 index YTD were shown as the slope in Table 2. In them the mean absolute value of the slope was $0.1371 \pm 0.1147$ (/year). In 17 correlations categorized in FIB4 index-increasing group, the mean value of the slope was $0.1212 \pm 0.1114$ (/year), yet in six ones categorized in FIB4 index-decreasing group, it was $-0.1823 \pm 0.1117$ (/year). Then, in a total of 41 correlations, the mean absolute value of the slope was $0.1764 \pm 0.1307$ (/year). In 22 positive correlations, the mean value of the slope was $0.1415 \pm 0.1118$ (/year), yet in 19 negative correlations, it was $-0.2168 \pm 0.1319$ (/year). All 41 correlations were shown in Figure 2. In order to demonstrate the slopes clearly, the main correlations were shown without $y$-intercept in Figure 2.

\section{Discussion}

In the present study the correlations between the period from the first to each examination and the mean FIB4 index YTD were analyzed. The results just would mean the time-dependent change of the mean FIB4 index YTD. All 23 enrolled patients had at least one phase with the main correlation (Figure 1 and Table 2) and the mean absolute value of $r$ was $0.91 \pm 0.08$. In 17 of these $23(74 \%)$ the absolute values of $r$ were over 0.90 . Meanwhile, 10 of 23 patients had only one phase with the main correlation (Figure 2(a)) and the remaining 13 had several phases (Figures 2(b)-2(d)). As a result, a total of 41 correlations were recognized in the study and the mean absolute value of $r$ was $0.92 \pm 0.07$. In 32 of all the 41 correlations $(78 \%)$ the absolute values of $r$ were over 0.90 (Table 3 ). In addition, the authors will show the reason why there were some correlations with low absolute values 


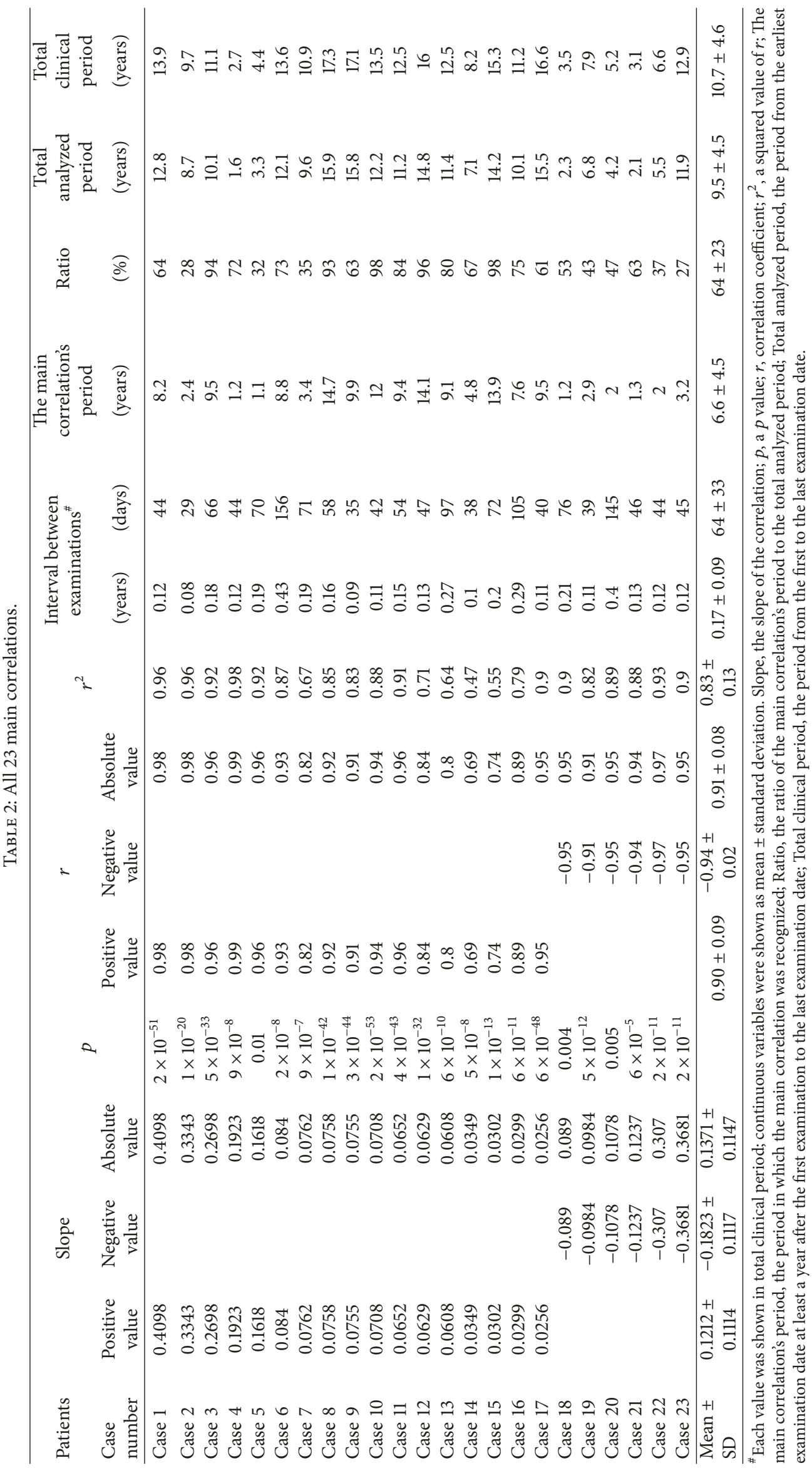




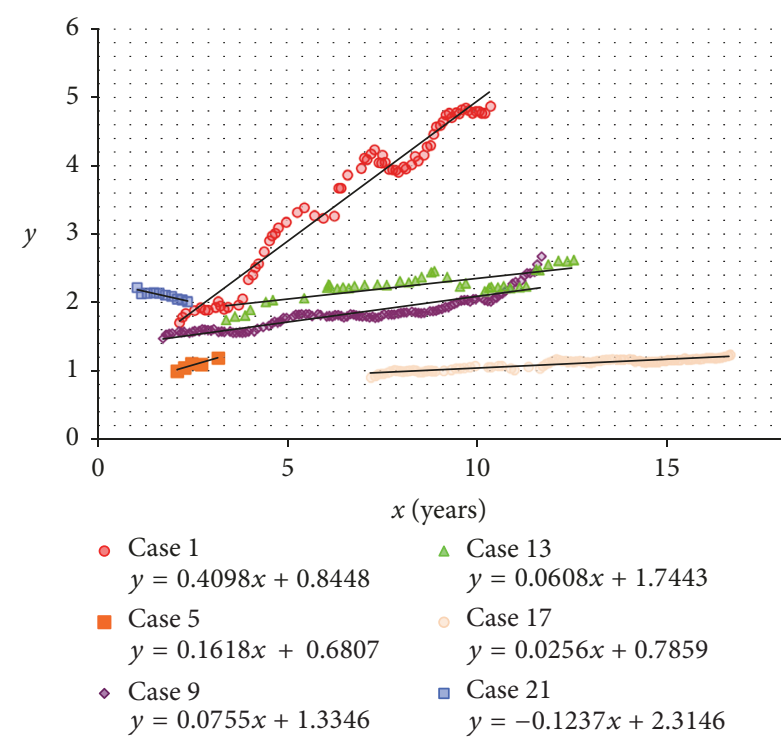

(a) The correlations in cases 1, 5, 9, 13, 17, and 21

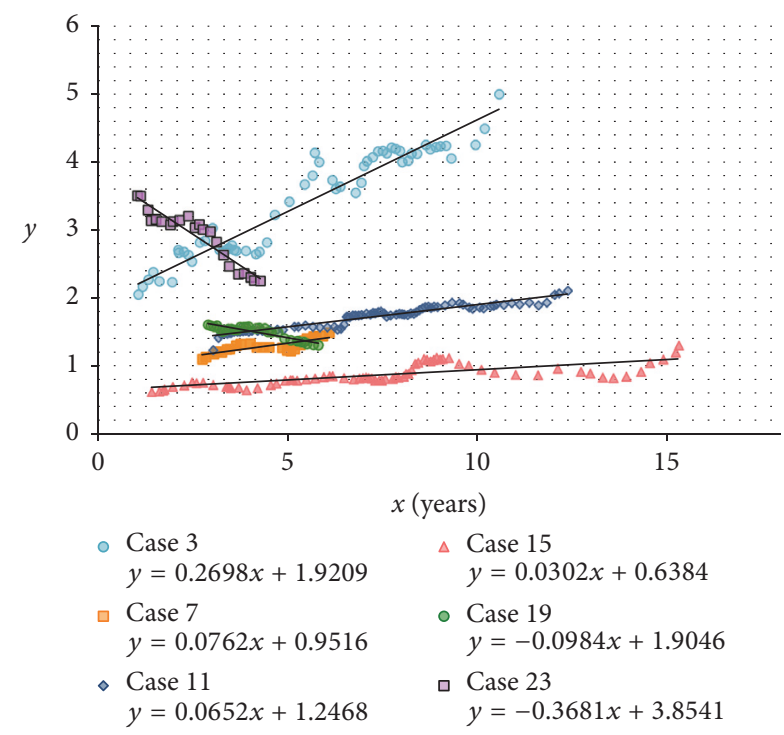

(c) The correlations in cases $3,7,11,15,19$, and 23

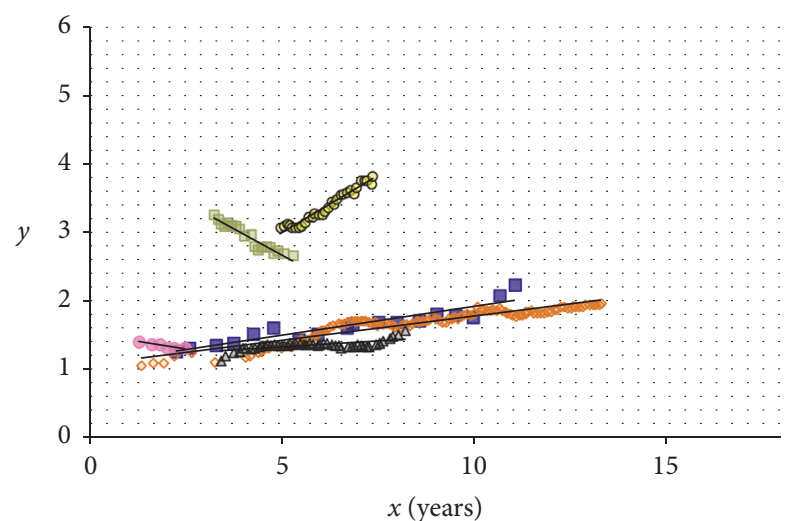

$$
\begin{array}{lc}
\triangle \text { Case } 2 & \Delta \text { Case } 14 \\
y=0.3343 x+1.3245 & y=0.0349 x+1.1499 \\
\square \text { Case } 6 & \circ \text { Case } 18 \\
y=0.0840 x+1.0741 & y=-0.0890 x+1.5139 \\
\diamond \text { Case } 10 & \square \text { Case } 22 \\
y=0.0708 x+1.0660 & y=-0.3070 x+4.1987
\end{array}
$$

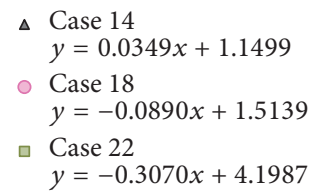

(b) The correlations in cases $2,6,10,14,18$, and 22

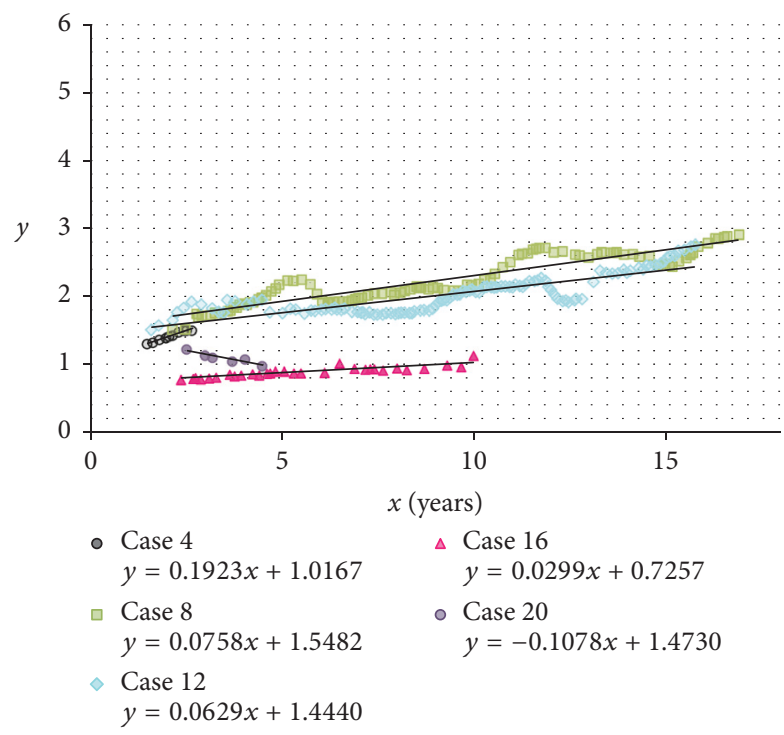

(d) The correlations in cases $4,8,12,16$, and 20

FIGURE 1: The correlation between the period from the first examination to each examination $(x)$ and the mean value of FIB4 index during the past year to each date of examination $(y)$ in the phase of all 23 main correlations.

of $r$. For example, out of all 41, three correlations (7.3\%) with the absolute values of $r$ less than 0.80 were recognized and all of them were the main correlations. They were enumerated in Figure 3. In all the three correlations the mean FIB4 index YTD gradually increased and then at once reached the peak, which was so-called "second peak point." After this point to the last point of data in the correlation, there was the bottom point, which was so-called "second bottom point." In these correlations the mean FIB4 index YTD moved like an italic type of " $N$." In such a condition, the absolute value of $r$ would probably become low.

In statistics, the coefficient of determination, which is calculated as a squared value of $r\left(r^{2}\right)$, determines how enough the outcomes could be explained by the hypotheses.
In this study, the mean value of $r^{2}$ in the main correlation was $0.83 \pm 0.13$ and that in a total of 41 correlations was $0.86 \pm 0.12$. The value of $r^{2}$ like these could not be ignored, even if the number was 23 or 41 . Moreover, it had been explained why there were some correlations with low absolute values of $r$. Statistically it was thought to be sufficient to speculate how the mean FIB4 index YTD moved.

There was another important thing about the movement of the mean FIB4 index YTD. In three of five patients with three phases (Figure 2(b)), the mean FIB4 index YTD showed decreasing firstly, increasing secondly, and decreasing finally, yet in two of these five (Figure 2(b)), it showed increasing, decreasing, and increasing. On the other hand, in eight patients with two phases (Figures 2(c)-2(d)), it showed firstly 


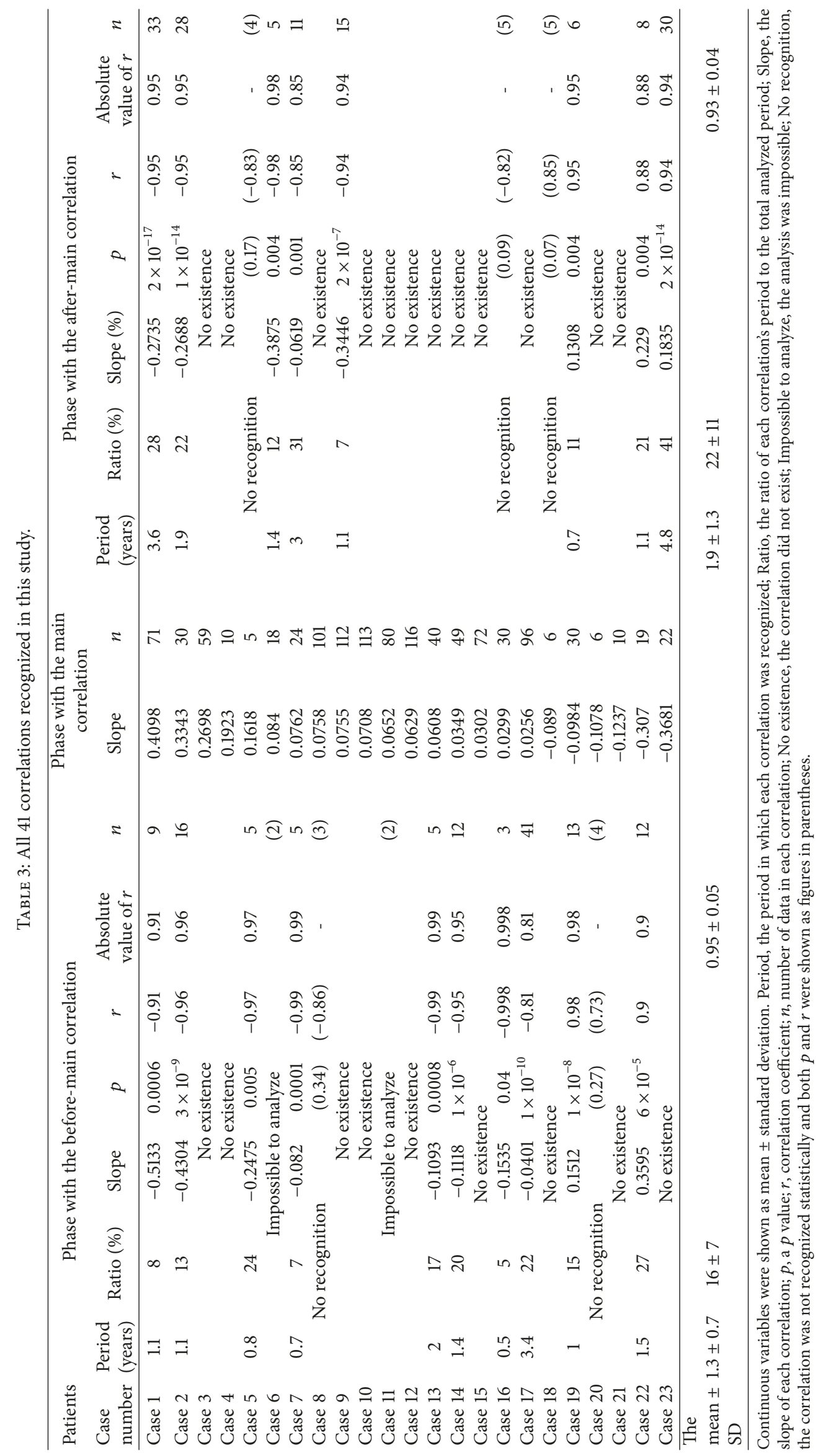




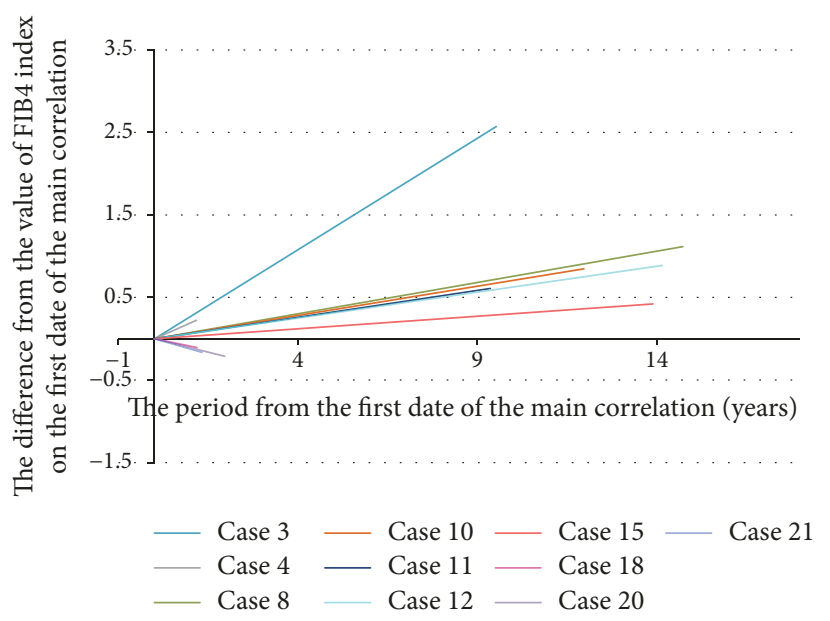

(a) In the patients who had only one phase with the main correlation

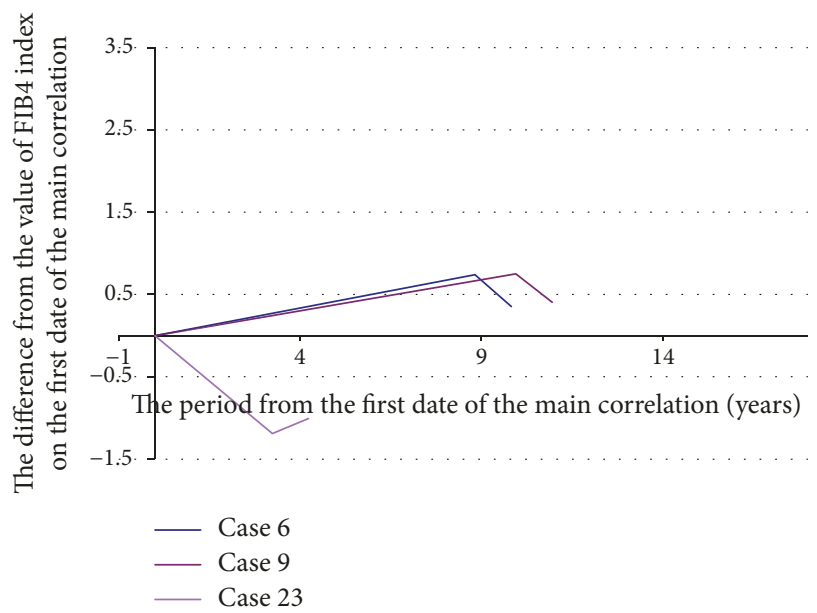

(c) In the patients who had two phases with the main and the after-main correlation

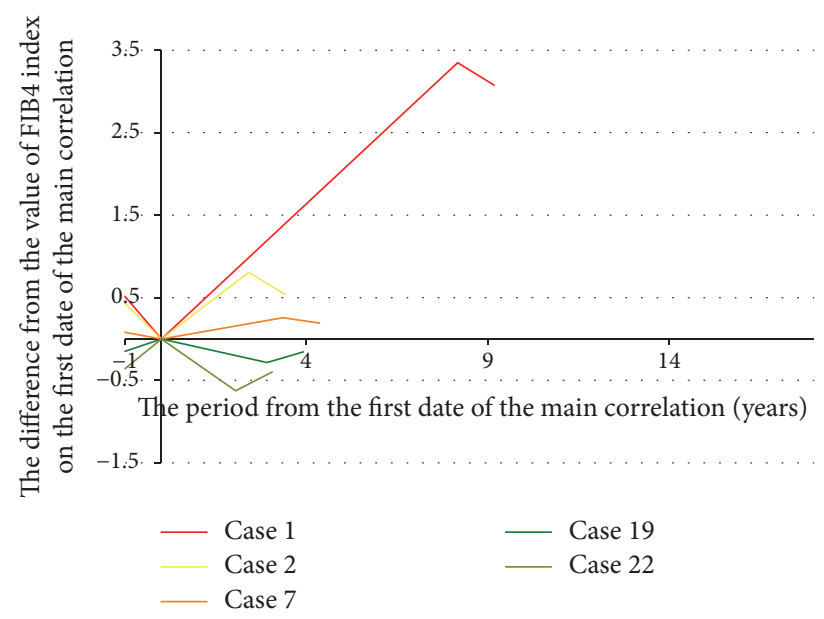

(b) In the patients who had three phases with the before-main, the main, and the after-main correlation

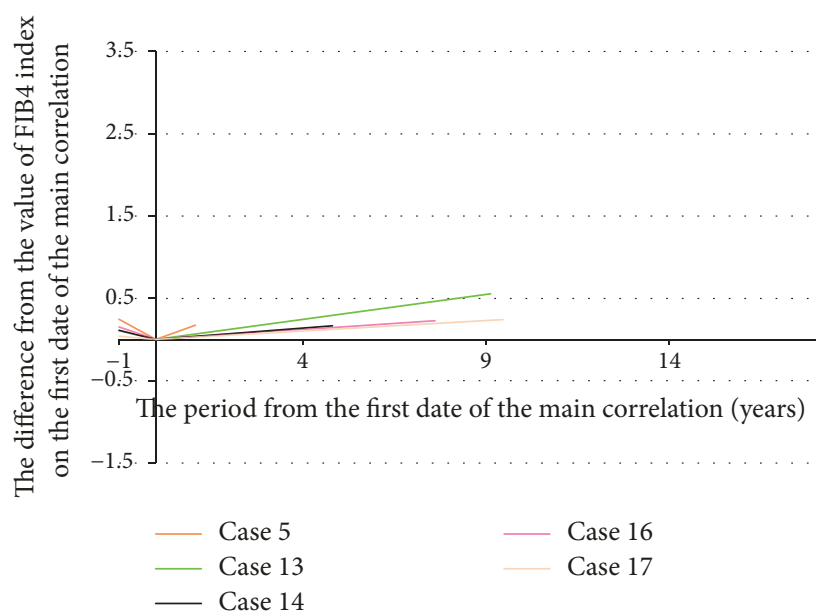

(d) In the patients who had two phases with the before-main and the main correlation

Figure 2: The slopes of all 41 correlations recognized in this study. All the main correlations were shown without $y$-intercept. (b, c, d): the before-main and the after-main correlations were shown only for a year.

decreasing and finally increasing or firstly increasing and finally decreasing. From the viewpoint of the movement, the most important thing was that there was a turning point in which the mean FIB4 index YTD changed from increasing to decreasing or from decreasing to increasing. This means that the mean FIB4 index YTD moved like a wave. Even in the main correlations, these waves were seen and the typical ones had been picked up in Figure 3.

Now, developing this study's methods, there would be a possibility. The possibility is that the methods will be applicable for any partial period. In order to validate it, the analysis only has to be performed, not from the first examination date and/or not to the last examination date. For example, in all the 23 patients the period was newly set from the closest date after half the total clinical period to that date after three-quarters. Limiting to this period, the analysis was newly performed through this study's methods. Out of 23 patients, seven whose analyzed period remained less than two years were excluded, because it was necessary for a year to calculate the mean FIB4 index YTD. In all the remaining 16 out of 23 , the new main correlations were analyzed. In the patient of case 16 there were only two pieces of data and it was impossible to analyze the correlation and in the patient of case 13 there were three pieces of data and the correlation was not statistically recognized by $p=0.08$ and $r=0.99$. However, in the remaining 14 patients the new main correlations were recognized and the mean absolute value of $r$ was $0.92 \pm 0.05$. In addition, in all 14, the absolute values of $r$ were over 0.80 . From this result, it was thought that the methods might be applicable for any partial period (Table 4).

The trigger of the start of the present study was a case report of a patient with NAFLD that the authors have already reported previously [26]. In this report we analyzed the correlation in a partial period between time (years) and the direct data of FIB4 index and we showed in that period that the direct data of FIB4 index decreased with the rate of 0.15 per year, statistically proven by the general linear regression model; FIB4 index $=4.90-0.15 \times$ time (years $)(p=0.02)$. 


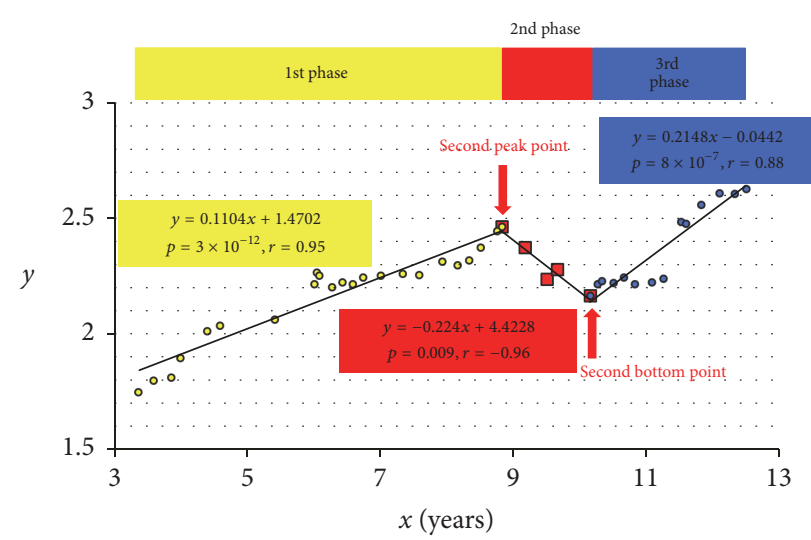

(a) The main correlation in case 13

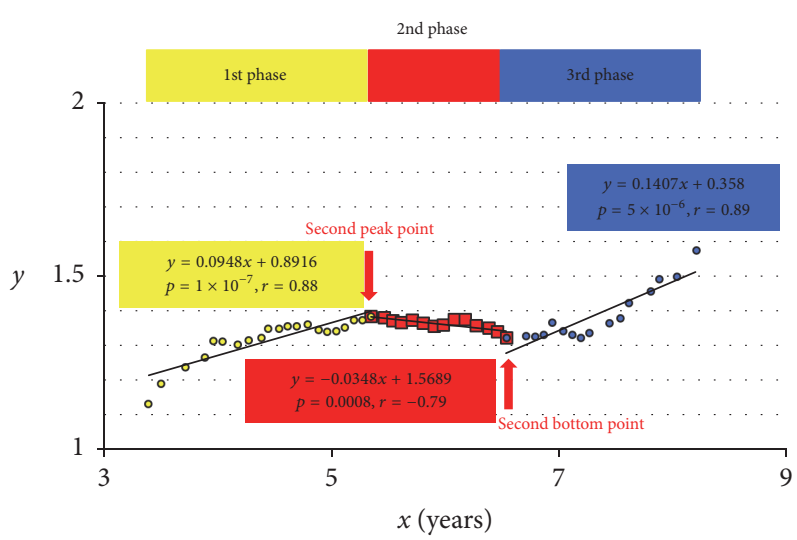

(b) The main correlation in case 14

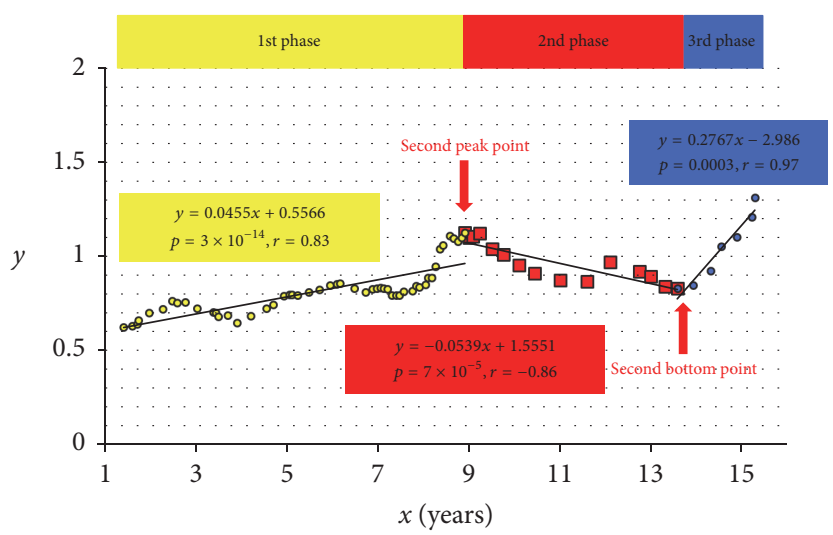

(c) The main correlation in case 15

FIGURE 3: All the three correlations with the absolute value of $r$ less than 0.80 . The value of $x$ was defined as the period from the first to each examination date and also the value of $y$ was defined as the mean value of FIB4 index during the past one year to each examination date. In all the three correlations, values of $y$ gradually increased and then at once reached the peak, which was so-called "second peak point." After this point to the last point of data in each correlation, there was the bottom point, which was so-called "second bottom point."

TABLE 4: All 14 new main correlations analyzed for the period newly set.

\begin{tabular}{|c|c|c|c|c|c|}
\hline Case number of the patient & Slope & Absolute value of slope & $p$ & Absolute value of $r$ & $n$ \\
\hline Case 1 & 0.415 & 0.415 & $6 \times 10^{-10}$ & 0.9 & 25 \\
\hline Case 2 & 0.4296 & 0.4296 & $9 \times 10^{-8}$ & 0.97 & 13 \\
\hline Case 3 & 0.6245 & 0.6245 & 0.001 & 0.89 & 9 \\
\hline Case 6 & 0.1186 & 0.1186 & 0.045 & 0.82 & 6 \\
\hline Case 7 & -0.1015 & 0.1015 & 0.035 & 0.9 & 5 \\
\hline Case 8 & 0.3461 & 0.3461 & $4 \times 10^{-11}$ & 0.99 & 13 \\
\hline Case 9 & 0.4383 & 0.4383 & $1 \times 10^{-12}$ & 0.99 & 16 \\
\hline Case 10 & 0.0921 & 0.0921 & $3 \times 10^{-9}$ & 0.89 & 25 \\
\hline Case 11 & 0.1192 & 0.1192 & $1 \times 10^{-10}$ & 0.96 & 19 \\
\hline Case 12 & 0.1062 & 0.1062 & $5 \times 10^{-16}$ & 0.95 & 31 \\
\hline Case 14 & -0.0458 & 0.0458 & 0.004 & 0.91 & 7 \\
\hline Case 15 & -0.1094 & 0.1094 & $8 \times 10^{-6}$ & 0.96 & 10 \\
\hline Case 17 & 0.1304 & 0.1304 & 0.004 & 0.88 & 8 \\
\hline Case 23 & 0.3707 & 0.3707 & 0.004 & 0.85 & 9 \\
\hline
\end{tabular}

The mean \pm SD $0.2462 \pm 0.1766$ $0.92 \pm 0.05$

Continuous variables were shown as mean \pm standard deviation. In all the 23 patients the period was newly set from the closest date after half the total clinical period to that date after three-quarters. Of 23 patients, seven whose analyzed period remained less than two years were excluded. In the remaining 16 patients, the new main correlations were analyzed. In two of these 16, the correlations were not recognized. In case 16 the correlation could not be analyzed because of only two data and in case 13 number of data was three and the correlation was not recognized by $p=0.08$ and $r=0.99$. Slope, the slope of the correlation; $p$, a $p$ value; $r$, correlation coefficient; $n$, number of data to analyze the correlation. 


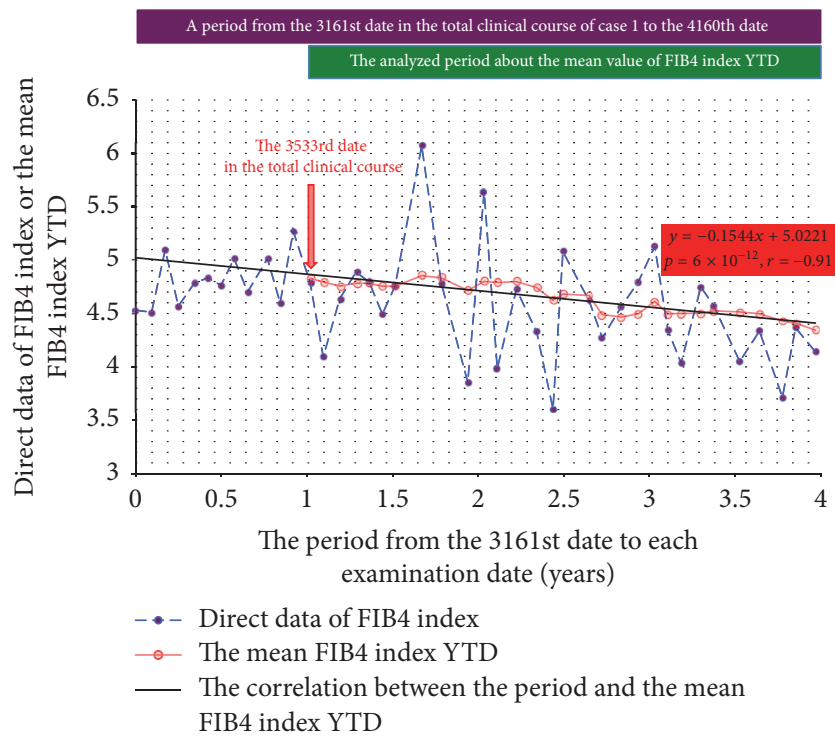

FIgURE 4: The transition of FIB4 index in a partial period in case 1. In a patient of case 1, a correlation was analyzed in a partial period newly set. The period was set from the 3161st date in the total clinical course to the 4610th date. After the earliest date more than a year from the 3161st date, that is, the 3533rd date finally to the 4160th date, the correlation between the period (years) from the 3161st date and the mean FIB4 index YTD was analyzed through this study's method. All the data were cited from our previous case report [26]. The mean FIB4 index YTD, the mean value of FIB4 index during the past one year.

This patient was enrolled as a patient of case 1 in the present study. If this study's methods were applicable for any partial period, similar outcome should be obtained. As expected, the mean FIB4 index YTD decreased with the rate of 0.15 per year (Figure 4); the mean FIB4 index YTD $=5.02-0.15 \times$ time (years) $\left(p=6 \times 10^{-12}\right.$ and $\left.r=-0.91\right)$. This was very similar to our previous formula and $p$ has become extremely low.

In this way, it was very easy to estimate increase-decrease rate per year of the mean FIB4 index YTD. It was the value of the slope of each correlation on the scatter diagram. As for the main correlations, the mean absolute rate per year was $0.1371 \pm 0.1147$ (Table 2). In this viewpoint, since the difference of FIB4 index between 1.30 and 2.67 [14, 27] is 1.37, it would take about 10 years by the mean absolute rate per year.

Meanwhile, the limitations of this study should be shown. Firstly, interval between examinations in this study was $0.17 \pm$ 0.09 (years) and it would be rather short. If the interval was longer, the dispersion of the data could not be minimized and the strong correlation might not be recognized. In fact, the after-main correlations or the before-main correlations were not recognized statistically in some patients, probably for the lack of number of data. About this, if the interval in the correlations had been shorter, the number of data would have increased and the correlation might have been seen. Anyway, when there were at least six pieces of data for the analysis, the correlations were all recognized in this study.
Secondly, it took a year from the first examination to calculate the data and also took another year to analyze the data.

Thirdly, in this study histological findings were not performed. Certainly, it was speculated that, compared to the earlier studies, liver fibrosis in the patients of this study would be rather advanced. Several proofs should be shown as follows. In this study the mean value of FIB4 index at the peak was $2.84 \pm 1.34$ (Table 1 ). Shah et al. reported that, for advanced fibrosis (stage 3-4), a FIB4 $>$ or $=2.67$ had an $80 \%$ positive predictive value [14]. Moreover, the mean value of platelet count at the bottom was $165 \pm 45\left(\times 10^{9} / \mathrm{L}\right)$ (Table 1). Kaneda et al. reported that the platelet count was found to be an independent predictor of cirrhosis and a cut-off value of $16 \times 10^{4} / \mathrm{microL}$ for the platelet count was associated with an optimal combination of sensitivity (100\%) and specificity (95\%) [8]. In addition, the mean value of type IV collagen $7 \mathrm{~S}$ at the peak was $5.2 \pm 2.0(\mathrm{ng} / \mathrm{mL})$ (Table 1$)$. It was reported that in patients with NASH the type IV collagen 7S domain was significantly elevated in patients with advanced fibrosis by multiple regression analysis [6] and Sumida et al. have developed NAFIC score in biopsy-proven patients with NAFLD to differentiate NASH from NAFLD, using the cut-off of type IV collagen $7 \mathrm{~S} \geq 5.0(\mathrm{ng} / \mathrm{mL})$ [7]. Then, the mean value of M2BPGi at the peak was $1.09 \pm 0.86$ (Table 1). Nishikawa et al. reported that in NASH patients the median values in each fibrosis stage were $0.7 \mathrm{COI}$ in F1, 0.7 COI in F2, 1.2 COI in F3, and 2.4 COI in F4 [28] and Lai et al. also reported that the AUROC of the COI for the diagnosis of fibrosis stages $\geq 1, \geq 2, \geq 3$, and 4 was $0.61,0.71,0.74$, and 0.84 , respectively [29]. These facts would show liver fibrosis in the enrolled patients would be rather advanced.

However, it is a problem whether the time-dependent change of FIB4 index corresponds to that of fibrosis by a liver biopsy. About this there was a retrospective study. McPherson et al. reported the following [30]. In 108 patients who had serial biopsies (median interval 6.6 years, range 1.3-22.6), there was a significant relationship between the change in fibrosis between biopsies and the change in both NAFLD fibrosis score [11] and FIB-4 score. They compared patients with histological evidence of increasing fibrosis stage (progressors) to subjects whose fibrosis remained stable or regressed (nonprogressors) and in progressors FIB-4 score was changed from $1.85 \pm 1.31$ at baseline biopsy to $2.33 \pm 1.69$ at follow-up one, yet in nonprogressors it was changed from $1.26 \pm 0.57$ to $1.36 \pm 0.62$ [30]

Nevertheless, there were few studies about the relationship between change in FIB4 index and change in fibrosis conducted by paired biopsies and therefore further verifications should be done. However, in the process of verifying it, this study's methods might be useful, because the dispersion of a single direct data of FIB4 index probably would be a considerable problem. It is difficult to perform biopsies to all patients with NAFLD and in such a condition the methods to minimize the dispersion of the data would be helpful.

To consider the risk of liver fibrosis based on grasping the whole picture of the movement of the mean FIB4 index YTD would be one of the practical benefits in the study. For all practical purposes, the latest correlation means either 
the main correlation or the after-main correlation and if the value of the slope of that correlation were positive, the progression of liver fibrosis would be concerned. Especially in a patient whose last data of the mean FIB4 index YTD in that correlation shows a value over 2.67 , advanced liver fibrosis should be well considered. On the other hand, in a patient with a negative value of the slope of the latest correlation, even if the last data of the mean FIB4 index YTD shows a value over 2.67 , it would be a little difficult to assess the risk. In fact, in a patient of case 1 , a sever complication was gradually improved in such a condition [26]. However, in such a patient careful treatment should be done to prevent the progression of liver fibrosis. Finally, if the value of the slope is negative and also the last data of the mean FIB4 index YTD is less than 1.30 in the latest correlation, the risk of liver fibrosis is considered to be low.

We hope that the methods in this study will be the benefits to patients with NAFLD and in the future the methods will be compared to other markers and modalities for estimating liver fibrosis, with increased number of patients.

\section{Conclusion}

This study demonstrated that in patients with NAFLD the correlations between the period from the first to each examination date and the mean value of FIB4 index during the past one year to each examination date were strongly recognized. Approximately, the time-dependent change of FIB4 index and its increase-decrease rate per year could be speculated simply and accurately.

\section{Conflicts of Interest}

The authors declare that there are no conflicts of interest regarding the publication of this paper.

\section{References}

[1] Z. M. Younossi, A. B. Koenig, D. Abdelatif, Y. Fazel, L. Henry, and M. Wymer, "Global epidemiology of nonalcoholic fatty liver disease-meta-analytic assessment of prevalence, incidence, and outcomes," Hepatology, vol. 64, no. 1, pp. 73-84, 2016.

[2] M. Ekstedt, H. Hagström, P. Nasr et al., "Fibrosis stage is the strongest predictor for disease-specific mortality in NAFLD after up to 33 years of follow-up," Hepatology, vol. 61, no. 5, pp. 1547-1554, 2015.

[3] E. H. Van Den Berg, M. Amini, T. C. M. A. Schreuder et al., "Prevalence and determinants of non-Alcoholic fatty liver disease in lifelines: A large Dutch population cohort," PLoS ONE, vol. 12, no. 2, Article ID e0171502, 2017.

[4] J. Uhanova, G. Minuk, F. Lopez Ficher, and N. Chandok, "Nonalcoholic Fatty Liver Disease in Canadian First Nations and Non-First Nations Patients," Canadian Journal of Gastroenterology and Hepatology, vol. 2016, Article ID 6420408, 2016.

[5] D. Kim and W. R. Kim, "Nonobese Fatty Liver Disease," Clinical Gastroenterology and Hepatology, vol. 15, no. 4, pp. 474-485, 2017.

[6] M. Yoneda, H. Mawatari, K. Fujita et al., "Type IV collagen 7s domain is an independent clinical marker of the severity of fibrosis in patients with nonalcoholic steatohepatitis before the cirrhotic stage," Journal of Gastroenterology, vol. 42, no. 5, pp. 375-381, 2007.

[7] Y. Sumida, M. Yoneda, H. Hyogo et al., "A simple clinical scoring system using ferritin, fasting insulin, and type IV collagen $7 \mathrm{~S}$ for predicting steatohepatitis in nonalcoholic fatty liver disease," Journal of Gastroenterology, vol. 46, no. 2, pp. 257-268, 2011.

[8] H. Kaneda, E. Hashimoto, S. Yatsuji, K. Tokushige, and K. Shiratori, "Hyaluronic acid levels can predict severe fibrosis and platelet counts can predict cirrhosis in patients with nonalcoholic fatty liver disease," Journal of Gastroenterology and Hepatology, vol. 21, no. 9, pp. 1459-1465, 2006.

[9] N. A. Palekar, R. Naus, S. P. Larson, J. Ward, and S. A. Harrison, "Clinical model for distinguishing nonalcoholic steatohepatitis from simple steatosis in patients with nonalcoholic fatty liver disease," Liver International, vol. 26, no. 2, pp. 151-156, 2006.

[10] P. Angulo, J. C. Keach, K. P. Batts, and K. D. Lindor, "Independent predictors of liver fibrosis in patients with nonalcoholic steatohepatitis," Hepatology, vol. 30, no. 6, pp. 1356-1362, 1999.

[11] P. Angulo, J. M. Hui, G. Marchesini et al., “The NAFLD fibrosis score: a noninvasive system that identifies liver fibrosis in patients with NAFLD," Hepatology, vol. 45, no. 4, pp. 846-854, 2007.

[12] S. A. Harrison, D. Oliver, H. L. Arnold, S. Gogia, and B. A. Neuschwander-Tetri, "Development and validation of a simple NAFLD clinical scoring system for identifying patients without advanced disease," Gut, vol. 57, no. 10, pp. 1441-1447, 2008.

[13] R. K. Sterling, E. Lissen, N. Clumeck et al., "Development of a simple noninvasive index to predict significant fibrosis in patients with HIV/HCV coinfection," Hepatology, vol. 43, no. 6, pp. 1317-1325, 2006.

[14] A. G. Shah, A. Lydecker, K. Murray, B. N. Tetri, M. J. Contos, and A. J. Sanyal, "Comparison of noninvasive markers of fibrosis in patients with nonalcoholic fatty liver disease," Clinical Gastroenterology and Hepatology, vol. 7, no. 10, pp. 1104-1112, 2009.

[15] S. McPherson, S. F. Stewart, E. Henderson, A. D. Burt, and C. P. Day, "Simple non-invasive fibrosis scoring systems can reliably exclude advanced fibrosis in patients with non-alcoholic fatty liver disease," Gut, vol. 59, no. 9, pp. 1265-1269, 2010.

[16] Y. Sumida, M. Yoneda, H. Hyogo et al., "Validation of the FIB4 index in a Japanese nonalcoholic fatty liver disease population," BMC Gastroenterology, vol. 12, article 2, 2012.

[17] A. Komori, A. Tanaka, H. Takikawa et al., "Guidelines for the management of primary biliary cirrhosis: The Intractable Hepatobiliary Disease Study Group supported by the Ministry of Health, Labour and Welfare of Japan," Hepatology Research, vol. 44, Supplement 1, pp. 71-90, 2014.

[18] R. Vuppalanchi, R. J. Gould, L. A. Wilson et al., "Clinical significance of serum autoantibodies in patients with NAFLD: Results from the nonalcoholic steatohepatitis clinical research network," Hepatology International, vol. 6, no. 1, pp. 379-385, 2012.

[19] S. S. Kunde, A. J. Lazenby, R. H. Clements, and G. A. Abrams, "Spectrum of NAFLD and diagnostic implications of the proposed new normal range for serum ALT in obese women," Hepatology, vol. 42, no. 3, pp. 650-656, 2005.

[20] A. L. Fracanzani, L. Valenti, E. Bugianesi et al., "Risk of severe liver disease in nonalcoholic fatty liver disease with normal aminotransferase levels: a role for insulin resistance and diabetes," Hepatology, vol. 48, no. 3, pp. 792-798, 2008.

[21] M. N. Kabbany, P. K. C. Selvakumar, K. Watt et al., "Prevalence of Nonalcoholic Steatohepatitis-Associated Cirrhosis in the United States: An Analysis of National Health and Nutrition 
Examination Survey Data," American Journal of Gastroenterology, vol. 112, no. 4, pp. 581-587, 2017.

[22] S. E. Mahady, P. Macaskill, J. C. Craig et al., "Diagnostic Accuracy of Noninvasive Fibrosis Scores in a Population of Individuals With a Low Prevalence of Fibrosis," Clinical Gastroenterology and Hepatology, vol. 15, no. 9, pp. 1453-1460.el, 2017.

[23] V. Ratziua, S. Bellentanib, and H. Cortez-Pintoc, "A position statement on NAFLD/NASH based on the EASL 2009 special conference," Journal of Hepatology, vol. 53, no. 2, pp. 372-384, 2010.

[24] European Association for the Study of the Liver (EASL), European Association for the Study of Diabetes (EASD), and European Association for the Study of Obesity (EASO), "EASL-EASD-EASO Clinical Practice Guidelines for the management of non-alcoholic fatty liver disease," Journal of Hepatology, vol. 64, no. 6, pp. 1388-1402, 2016.

[25] E. M. Hennes, M. Zeniya, A. J. Czaja et al., "Simplified criteria for the diagnosis of autoimmune hepatitis," Hepatology, vol. 48, no. 1, pp. 169-176, 2008.

[26] H. Miyata and S. Miyata, "A case of hepatopulmonary syndrome derived from nonalcoholic fatty liver disease with severe liver fibrosis, in which hypoxia could be recovered by the improvement of liver fibrosis," Kanzo (Acta Hepatologica Japonica), vol. 55, no. 8, pp. 479-487, 2014.

[27] T. Wada and M. Zeniya, "Background of the FIB-4 index in japanese non-alcoholic fatty liver disease," Internal Medicine, vol. 54, no. 2, pp. 127-132, 2015.

[28] H. Nishikawa, H. Enomoto, Y. Iwata et al., "Clinical significance of serum Wisteria floribunda agglutinin positive Mac-2binding protein level in non-alcoholic steatohepatitis," Hepatology Research, vol. 46, no. 12, pp. 1194-1202, 2016.

[29] L.-L. Lai, W.-K. Chan, P. Sthaneshwar, N. R. N. Mustapha, K.-L. Goh, and S. Mahadeva, "Serum Wisteria floribunda agglutininpositive Mac-2 binding protein in non-alcoholic fatty liver disease," PLoS ONE, vol. 12, no. 4, Article ID e0174982, 2017.

[30] S. McPherson, T. Hardy, E. Henderson, A. D. Burt, C. P. Day, and Q. M. Anstee, "Evidence of NAFLD progression from steatosis to fibrosing-steatohepatitis using paired biopsies: implications for prognosis and clinical management," Journal of Hepatology, vol. 62 , no. 5, pp. 1148-1155, 2015. 


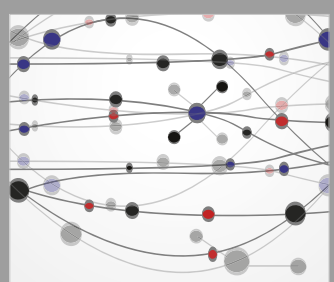

The Scientific World Journal
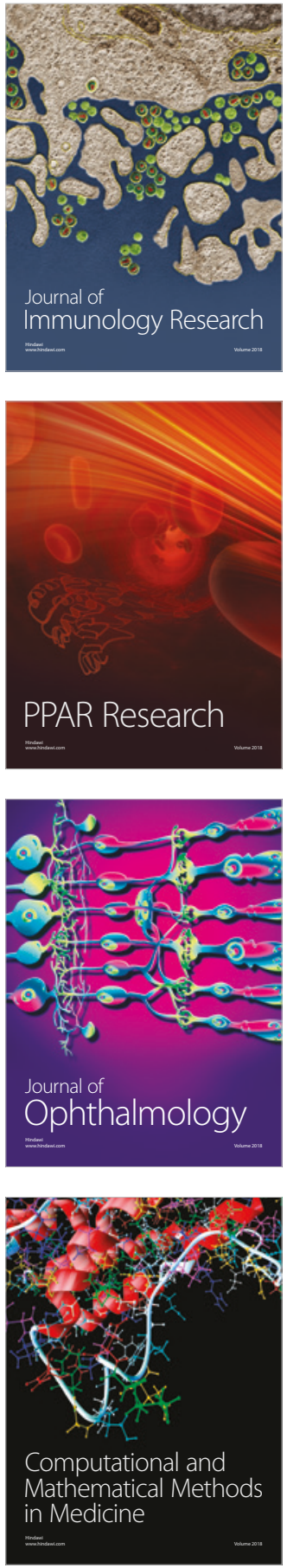

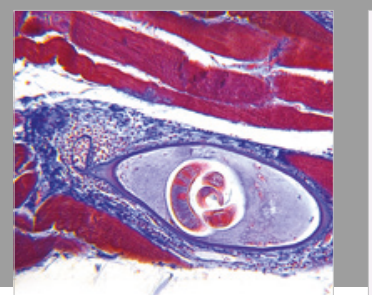

Gastroenterology Research and Practice

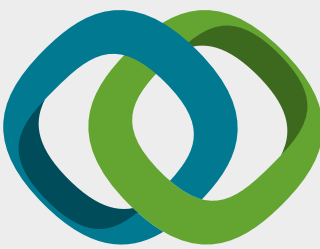

\section{Hindawi}

Submit your manuscripts at

www.hindawi.com
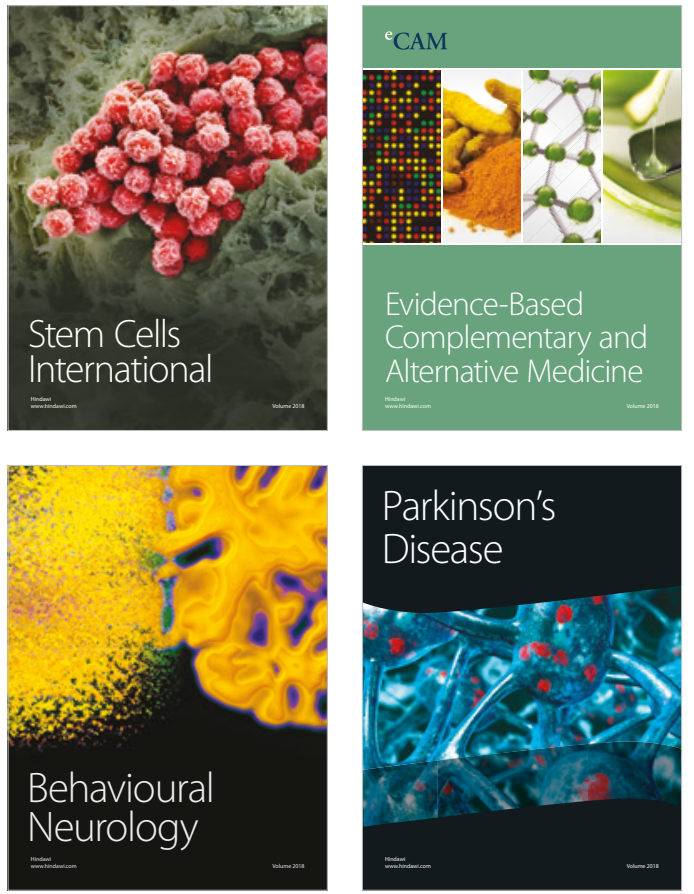

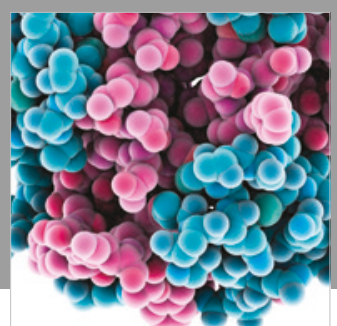

ournal of

Diabetes Research

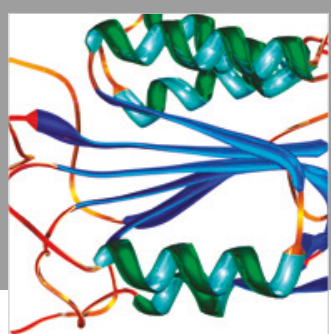

Disease Markers
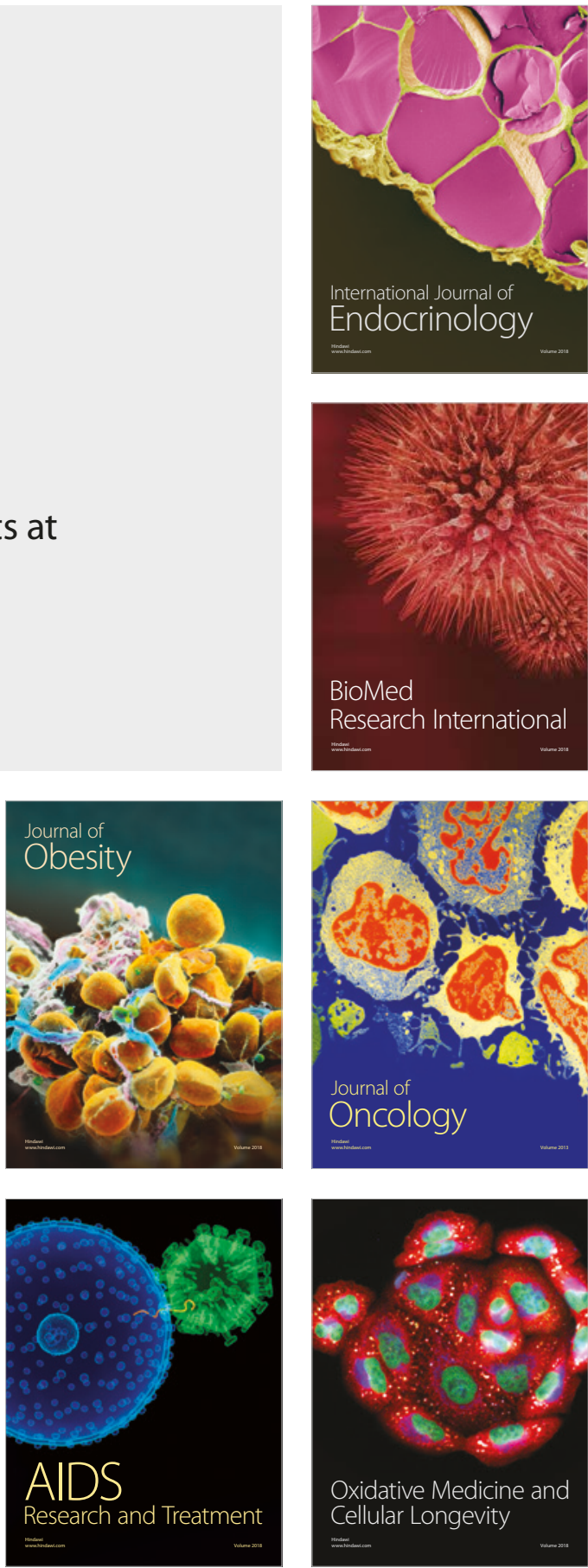